\title{
AUTHOR INDEX \\ VOLUME 41 (2015)
}

Anjum, A. A., see Tahir, R.

Chan, F.-T., see Chiou, H.-Y.

Chang, H.-H., see Kuo, T.-F.

Chang, H.-W., see Chiou, H.-Y.

Chang, L.-J., see Chiou, H.-Y.

Chang, M.-H., see Wang, C.-Y.

Chang, M.-H., see Wang, C.-Y.

Chang, P.-C., see Li, K.-P.

Chang, P.-C., see Yang, C.-H.

Chang, P.-C., see Yen, T.-Y.

Chang, P.-H., see Wang, F.-I.

Chang, T.-C., see Tsai, S.-S.

Chang, T.-H., see Wang, C.-Y.

Chang, T.-H., see Wang, C.-Y.

Chen, C.-L., see Lee, Y.-L.

Chen, C.-L., Wang, S.-T., Chu, C. and Wang, S.-H., Comparison of Four Molecular Typing Methods for Riemerella anatipestifer

Chen, H.-T., Wu, H.-Y., Shih, C.-H. and Jan, T.-R., A Differential Effect of Graphene

Oxide on the Production of Proinflammatory Cytokines by Murine Microglia

Chen, J.-L., see Wang, F.-I.

Chen, L.-J., see Tsai, S.-S.

Chen, M.-H., see Kuo, T.-F.

Chen, P.-H., see Wang, C.-Y.

Chen, P.-H., see Wang, C.-Y.

Chen, R.-S., see Kuo, T.-F.

Chen, S.-T., see Kuo, T.-F.

Chen, Y.-N., Wu, C. C. and Lin, T. L., Turkey Coronavirus: An Updated Review

Cheng, C.-H., see Wang, P.-Y.

Chi, C.-H., see Lee, Y.-L.

Chiou, H.-Y., Yeh, K.-S., Jeng, C.-R., Chang, H.-W., Chang, L.-J., Wu, Y.-H., Chan, F.-T. and Pang, V. F., Disease Surveillance in Rescued and Road-Killed Wild-Ranging

Carnivores in Taiwan

Chiou, M.-T., see Tsai, S.-S.

Chu, C., see Chen, C.-L.

Chuang, K. P., see Tsai, S.-S.

Hou, W.-C., see Wang, P.-Y.

Hsieh, C.-H., see Kuo, T.-F.

Hsu, S.-L., see Yang, C.-H.

$\mathrm{Hu}$, S.-C., see Yang, J.-L.

Huang, D., see Wang, L.-C.

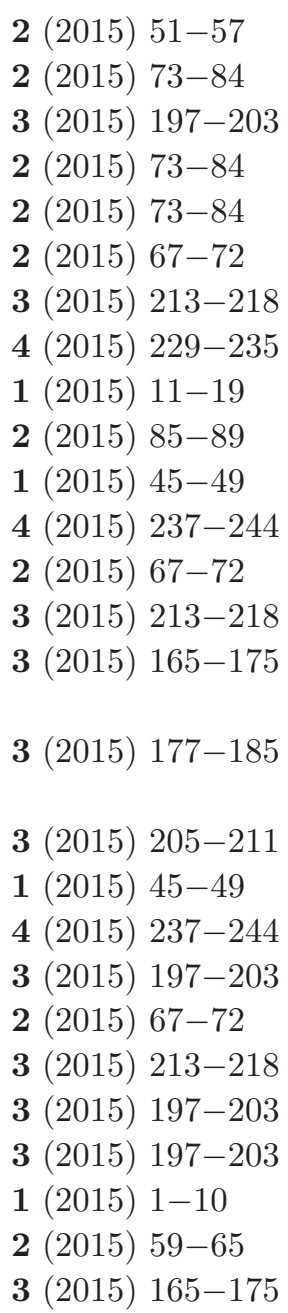

2 (2015) 73-84

4 (2015) 237-244

3 (2015) 177-185

4 (2015) $237-244$

2 (2015) 59-65

3 (2015) 197-203

1 (2015) 11-19

3 (2015) 187-196

4 (2015) 251-255 
Hung, S. Y. and Wang, F.-I., Impairment of Non-Phagocytosis-Associated Oxidative Burst to Actinobacillus pleuropneumoniae in Porcine Neutrophils Induced by Pseudorabies Virus Jan, T.-R., see Wang, P.-Y.

Jan, T.-R., see Chen, H.-T.

Jeng, C.-R., see Wang, F.-I.

Jeng, C.-R., see Chiou, H.-Y.

Jiang, C.-C., see Kuo, T.-F.

Jong, T.-T., see Yang, C.-H.

Khan, F., see Tahir, R.

Kuo, T.-F., see Wang, C.-Y.

Kuo, T.-F., see Wang, C.-Y.

Kuo, T.-F., Sheu, S.-Y., Jiang, C.-C., Chang, H.-H., Chen, S.-T., Chen, R.-S., Hsieh, C.-H. and Chen, M.-H., Tooth Regeneration with Dental Stem Cell Research in Miniature Pig Model

Lai, S. Y.-S., see Wang, F.-I.

Lee, F., see Yang, J.-L.

Lee, J.-J., see Wang, S.-L.

Lee, L. H., see Wu, H. C.

Lee, Y.-L., Yu, P.-H., Chen, C.-L., Wu, Y.-L. and Chi, C.-H., Determination of the Enhancement Effect and Diameters of the Major Arteries of African Grey Parrots Using a Dual-Head Power Injector for Computed Tomographic Angiography

Lei, Y.-C., see Wang, C.-Y.

Li, K.-P., see Yen, T.-Y.

Li, K.-P., Ou, S.-C., Shien, J.-H. and Chang, P.-C., Detection and Differentiation of the Vaccine Strain and Field Isolates of Duck Hepatitis A Virus Type 1 Using Real-Time RT-PCR and High Resolution Melting Assays

Li, Y.-C., see Yang, J.-L.

Liao, A. T., see Wang, S.-L.

Lin, C. C., see Wu, H. C.

Lin, D.-Y., see Wang, L.-C.

Lin, T. L., see Chen, Y.-N.

Lin, T. L., see Mosley, Y.-Y. C.

Liu, C.-H., see Wang, F.-I.

Liu, P.-C. and Su, B.-L., Causes of Canine Anemia in Taiwan: A Five-Year Retrospective Survey

Mosley, Y.-Y. C., Wu, C. C. and Lin, T. L., Avian Viral Vector Vaccines for Infectious

Bursal Disease

Muhammad, K., see Tahir, R.

Ou, S.-C., see Yen, T.-Y.

Ou, S.-C., see Li, K.-P.

Pang, V. F., see Wang, F.-I.

Pang, V. F., see Chiou, H.-Y.

Rasool, A., see Tahir, R.

Shen, P. C., see Wu, H. C.

Sheu, S.-Y., see Kuo, T.-F.

Sheu, S.-Y., see Wang, C.-Y.

Sheu, S.-Y., see Wang, C.-Y.

Shien, J.-H., see Li, K.-P.

Shien, J.-H., see Yen, T.-Y.

Shih, C.-H., see Chen, H.-T.

1 (2015) 21-29

2 (2015) 59-65

3 (2015) 205-211

1 (2015) 45-49

2 (2015) 73-84

3 (2015) 197-203

1 (2015) 11-19

2 (2015) 51-57

2 (2015) 67-72

3 (2015) 213-218

3 (2015) 197-203

1 (2015) 45-49

3 (2015) 187-196

1 (2015) 39-43

4 (2015) 219-228

3 (2015) 165-175

3 (2015) 213-218

2 (2015) 85-89

4 (2015) 229-235

3 (2015) 187-196

1 (2015) 39-43

4 (2015) 219-228

4 (2015) 245-249

1 (2015) 1-10

3 (2015) 153-163

1 (2015) 45-49

1 (2015) 31-37

3 (2015) 153-163

2 (2015) 51-57

2 (2015) 85-89

4 (2015) 229-235

1 (2015) 45-49

2 (2015) 73-84

2 (2015) 51-57

4 (2015) 219-228

3 (2015) 197-203

2 (2015) 67-72

3 (2015) 213-218

4 (2015) 229-235

2 (2015) 85-89

3 (2015) 205-211 
Shih, C.-Y., see Tsai, S.-S.

4 (2015) $237-244$

$\mathrm{Su}, \mathrm{B} . \mathrm{S}$. , see $\mathrm{Wu}, \mathrm{H} . \mathrm{C}$.

$\mathrm{Su}$, B.-L., see Liu, P.-C.

Tahir, R., Anjum, A. A., Muhammad, K., Rasool, A. and Khan, F., Avian Influenza and Its

Mass Depopulation Strategies in Infected Poultry Birds

Tan, D.-H., see Yang, C.-H.

Thong, W., see Wang, L.-C.

Tsai, S.-S., Chen, L.-J., Shih, C.-Y., Chang, T.-C., Chiou, M.-T. and Chuang, K. P., Joint Lesions in Taiwan Native Colored Broiler Chicken with Natural and Experimental

Staphylococcus aureus or S. cohnii Infection

Wang, C.-H., see Wang, L.-C.

Wang, C.-Y., Sheu, S.-Y., Lei, Y.-C., Wang, J.-H., Chang, M.-H., Chen, P.-H., Chang,

T.-H. and Kuo, T.-F., Evaluation of Amoxicillin Withdrawal Period Time in Pompano

Liver and Kidney

Wang, C.-Y., Sheu, S.-Y., Wang, J.-H., Chang, M.-H., Chen, P.-H., Chang, T.-H. and Kuo,

T.-F., Estimation of the Withdrawal Period for Amoxicillin in Pompano (Trachinotus

blochii) Serum and Muscle

Wang, F.-I., see Hung, S. Y.

Wang, F.-I., see Yang, J.-L.

Wang, F.-I., Yu, J.-F., Chen, J.-L., Lai, S. Y.-S., Jeng, C.-R., Liu, C.-H., Chang, P.-H. and

Pang, V. F., Case Report: A Primary Splenic Fibrosarcoma with Hepatic Metastasis in a

Captive Koala (Phascolarctos cinereus)

Wang, J.-H., see Wang, C.-Y.

Wang, J.-H., see Wang, C.-Y.

Wang, L.-C. and Huang, D., Highly Sensitive H7N9 Avian Influenza Virus Detection Using

Reverse Transcription Loop-Mediated Isothermal Amplification and Oligonucleotide

Microarray

Wang, L.-C., Lin, D.-Y., Thong, W. and Wang, C.-H., Multiplex Reverse Transcription

Polymerase Chain Reaction for Chicken Tumor Virus Detection

Wang, P.-Y., Wu, H.-Y., Cheng, C.-H., Hou, W.-C. and Jan, T.-R., Hispolon Differentially

Modulated the Production of Antigen-Induced T Cell Cytokines via the Regulation of

Cellular Glutathione

Wang, S.-H., see Chen, C.-L.

Wang, S.-L., Lee, J.-J. and Liao, A. T., Case Report: Low Dose Cyclosporine Combined

Ketoconazole Cures a Dog of Sterile Nodular Panniculitis

Wang, S.-T., see Chen, C.-L.

Wen, C.-L., see Yang, C.-H.

Wu, C. C., see Chen, Y.-N.

Wu, C. C., see Mosley, Y.-Y. C.

Wu, H. C., Shen, P. C., Lin, C. C., Su, B. S. and Lee, L. H., The In Vitro and In Vivo

Cytotoxicity of Liposome-Encapsulated Dichloromethylene-Diphosphonate on Chicken

Macrophages and the Potential of Using Macrophage-Depleted Chickens as a Model for

Reovirus Infection

Wu, H.-Y., see Chen, H.-T.

Wu, H.-Y., see Wang, P.-Y.

Wu, Y.-H., see Chiou, H.-Y.

Wu, Y.-L., see Lee, Y.-L.

Yang, C.-H., Tan, D.-H., Jong, T.-T., Wen, C.-L., Hsu, S.-L. and Chang, P.-C., In Vitro

Anti-Viral Activity of Ethanol Extract from Ixeris chinensis Against Influenza Virus

4 (2015) 219-228

1 (2015) 31-37

2 (2015) 51-57

1 (2015) 11-19

4 (2015) 245-249

4 (2015) 237-244

4 (2015) 245-249

3 (2015) 213-218

2 (2015) $67-72$

1 (2015) 21-29

3 (2015) 187-196

1 (2015) 45-49

2 (2015) 67-72

3 (2015) 213-218

4 (2015) 251-255

4 (2015) 245-249

2 (2015) 59-65

3 (2015) 177-185

1 (2015) 39-43

3 (2015) 177-185

1 (2015) 11-19

1 (2015) 1-10

3 (2015) 153-163

4 (2015) 219-228

3 (2015) 205-211

2 (2015) 59-65

2 (2015) 73-84

3 (2015) 165-175

1 (2015) 11-19 
Yang, J.-L., Li, Y.-C., Hu, S.-C., Lee, F. and Wang, F.-I., Rapid Diagnosis of Bluetongue Virus Serotypes 2 and 12 Infection by Reverse Transcription Loop-Mediated Isothermal Amplification

Yeh, K.-S., see Chiou, H.-Y.

Yen, T.-Y., Li, K.-P., Ou, S.-C., Shien, J.-H. and Chang, P.-C., The White Roman Goose as a Host for Infection and Viral Shedding of Muscovy Duck Parvovirus

Yu, J.-F., see Wang, F.-I.

Yu, P.-H., see Lee, Y.-L.
3 (2015) $187-196$

2 (2015) 73-84

2 (2015) 85-89

1 (2015) 45-49

3 (2015) 165-175 